\title{
Development of a magnetic tracking system for monitoring soil movements induced by geohazards
}

\author{
Xiaoyu Chen $^{1, *}$ and Rolando P. Orense ${ }^{1}$ \\ ${ }^{1}$ Department of Civil and Environmental Engineering, University of Auckland, Auckland, NZ
}

\begin{abstract}
In the study of geotechnical hazards, such as soil liquefaction and landslides, the analysis of soil movements is always one of the major preoccupations. An efficient movement sensing technique requires the tracking of subsurface soil for the purpose of examining the mechanism involved. A magnetic tracking system is therefore proposed, with permanent magnets as trackers and magnetometers as receivers. When permanent magnets, deployed within the soil to serve as excitation sources, move with soil body during a geotechnical event, they generate static magnetic fields whose flux densities are related with the positions and orientations of the magnets. Magnetometers are used as receivers to detect the generated magnetic fields, which can be further used in calculating the magnets' locations and orientations based on appropriately developed algorithms. Comparison between situations where the trackers are exposed to air and embedded within soil was conducted to evaluate the influence of soil (wet and dry) on the tracking accuracy. Also, multi-objective tracking is realized by using the particle swarm optimization (PSO) technique combined with interior-point algorithm. The tracking errors are evaluated and applications of the proposed system in small-scale laboratory tests for geohazards are discussed.
\end{abstract}

\section{Introduction}

Economic cost and human loss from geohazards, such as liquefaction and landslides, lead to the needs for mitigation methods in order to reduce the risks of such hazards. Identification of possible failure mechanisms and assessment of potential damage associated with earthquake hazards are important ingredients in mitigating their impacts to the built environment [1]. Studying the movement properties during geohazards may help researchers have a better understanding of the mechanism involved in landslides and soil liquefaction, or at least, be used in a monitoring or an early warning system. More specifically, for an early warning system on landslides, it is very effective to include a movement sensing system simply because large movement of soil bodies is one of the most direct signs to indicate a potential failure. No matter what complex mechanisms are behind, these geohazards, such as landslides and soil liquefaction, manifest themselves with soil movements or the potential of soil movements. For example, liquefaction-induced ground movements have been given special attentions by the geotechnical engineering community. Field observations are considered straightforward information and can be used to verify some predictive models, but a shortcoming is that subsurface deformations are usually beyond observations. Besides, laboratory tests, such as flume tests, shaking table tests and centrifuge tests, in which the influencing factors, initial and boundary conditions can be tightly controlled, have shown great potential and have already been conducted by many researchers using various novel techniques. Baba et al. [2] investigated soil creep movements along slopes by measuring velocity of soil particles non-invasively using particle image velocimetry (PIV), which is a type of 2D image analysis technique widely accepted by geotechnical engineers as a deformation measurement method. Take et al. [3] used digital image correlation (DIC) method to study the failure mechanism of a shallow foundation. The principle of image-based deformation measurement is to match the patterns of soil particles between two successive pictures within a geotechnical process. Consequently, all local displacement vectors representing the movements of soil particles can be visualised by incremental pixel movements. The noninvasive $2 \mathrm{D}$ image technique is able to detect the movement of soil particles down to $\mu \mathrm{m}$. Additionally, the advances in camera hardware will result in direct improvements in the accuracy and precision of DIC. Maintaining the natural texture of soil particles without changing the chemical or mechanical properties of soil is another advantage of 2D image analysis. However, one of the drawbacks of the image-based technique is that interface friction between soil and transparent plate can extensively influence the behaviour of soil at the boundary of the physical tests. Secondly, while DIC technique is nowadays used successfully in analysis of planar (2D) deformation, in the study of liquefaction or other geotechnical procedures, spatial movements within

* Corresponding author: xche753@aucklanduni.ac.nz 
soil bodies gain more importance. In 2008, by increasing the number of cameras from one set to two orthogonally placed to each other, McLeod [4] was able to acquire field measurements of surface heave during pipe bursting. The application of DIC provided a $2.5 \mathrm{D}$ deformation pattern on the ground surface during the underground construction process. Although a $2.5 \mathrm{D}$ deformation was captured, big concerns still exist since it was only possible to capture the movements of the soil at surface, while the behaviour of soil within the soil body was beyond recognition.

In order to overcome the shortcomings described above, this paper aims to present a novel technique which is able to measure subsurface deformation of soil by deploying trackers underground, whose $3 \mathrm{D}$ positions and $2 \mathrm{D}$ orientations can be captured by a sensor array established outside a soil body. Firstly, necessary mathematical background of the magnetic tracking system is discussed. Secondly, a verification test is presented evaluating the accuracy of the system when used in usual geotechnical environment, i.e. with the presence of soil (wet and dry). Finally, multi-objective tracking is tested and errors are analysed.

\section{Magnetic tracking system}

\subsection{Background}

Magnetic sensor system is a type of positioning sensing technique with an obvious advantage of non-contact operation. A magnetic dipole, such as a permanent magnet or a coil emitting electromagnetic signals, can generate static magnetic field in the space around it. Position as well as orientation information can be derived by measuring the magnetic flux density ( $\boldsymbol{B}$ with the unit of Tesla) around the magnetic dipole. Magnetometers are usually used to detect the magnetic flux density. The magnetic tracking technique has already been used outside geotechnical engineering. For example, Schlageter et al. [5] developed a system capable of tracking a permanent magnet with a 2D-array of 16 cylindrical Hall sensors. Hu et al. [6] investigated the use of magnet-based localization in wireless capsule endoscopic technique.

Soil has a magnetic permeability very similar to that of non-ferromagnetic materials, such as air and water, and therefore it cannot influence the static magnetic field generated by magnets, as indicated by Fialová et al. [7]. Therefore, it is possible to localize magnetic trackers buried in soil with high accuracy. Magnetic flux density at a certain target point in the space gives information about the relative position of the magnetic tracker to that target point. As soil moves due to geohazards, the magnetic tracker will move along with the soil body. In this way, the movements of soil can be illustrated by the movements of the magnetic tracker.

\subsection{Mathematical model}

Magnetic flux density detected by magnetometer array is used to calculate the $3 \mathrm{D}$ positions and $2 \mathrm{D}$ orientations of a tracker. By using a permanent magnet as tracker, a convenient approximation is to consider the magnet as a point dipole whose largest dimension is much smaller than the distance between the tracker and the magnetometer array. As shown in Figure 1, the relationship between the tracker's 3D position and 2D orientation and its magnetic flux density detected by the array is given by Equations (1) - (4).

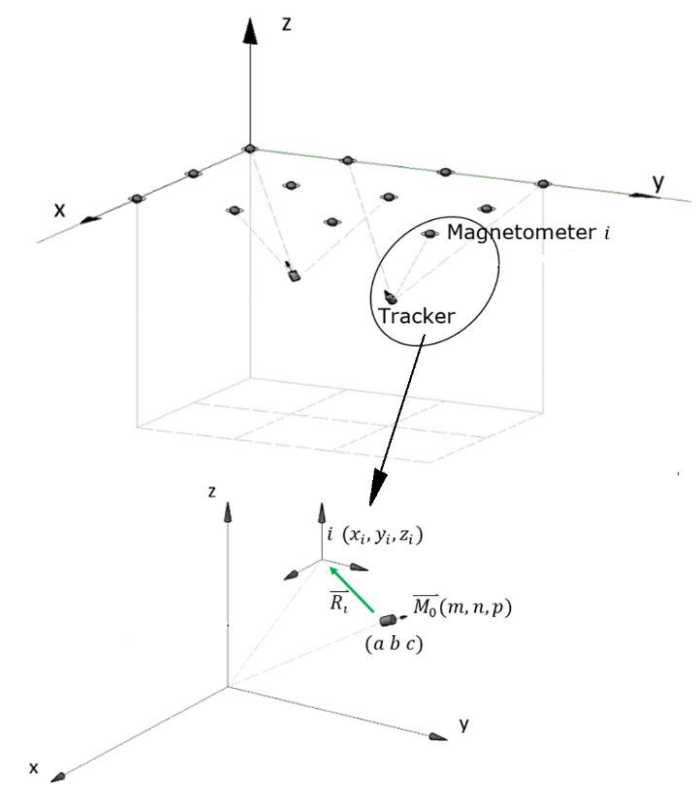

Fig. 1. Schematic of the magnetic sensor system in the $3 D$ Cartesian coordinate system.

$$
\begin{gathered}
\boldsymbol{B}_{i}=B_{T}\left(\frac{3\left(M_{0} \cdot R_{i}\right) \cdot R_{i}}{R_{i}^{5}}-\frac{M_{0}}{R_{i}^{3}}\right) \\
R_{i}=\sqrt{\left(x_{i}-a\right)^{2}+\left(y_{i}-b\right)^{2}+\left(z_{i}-c\right)^{2}} \\
\boldsymbol{R}_{i}=\left(x_{i}-a, y_{i}-b, z_{i}-c\right) \\
\boldsymbol{M}_{0}=(m, n, p)
\end{gathered}
$$

where $(a, b, c)$ denotes the 3 unknown locations on the three orthogonal axes of the 3D Cartesian coordinate system, and $(m, n, p)$ denote the orientations with the relationship $m^{2}+n^{2}+p^{2}=1$ holds; $B_{T}$ is a constant parameter related to the magnet being used; $\boldsymbol{B}_{\boldsymbol{i}}$ is the magnetic flux density detected by magnetometer $i$, where the location of the magnetometer $\boldsymbol{i}$ is indicated by $\left(x_{i}, y_{i}, z_{i}\right) ; \boldsymbol{M}_{0}$ is a unit vector which indicates the direction of the magnetic dipole, as shown in Figure 1. Further discussions of the algorithm developed and validation using finite element method magnetics are presented by Chen and Orense [8].

\section{Verification tests}

Verification tests were performed for evaluating the influence of the presence of soil (both dry and wet) on tracking accuracy considering its future applications under geotechnical environments. Some parameters 
related with the hardware being used in the tracking system should be noted in advance.

Neodymium is considered to be the strongest available magnet material, which is therefore used in the tracking system. The size of the magnet used was $15 \mathrm{~mm}$ in diameter and $20 \mathrm{~mm}$ in height with a residual magnetism (Br) of 1.32-1.37 Tesla. The magnet was covered by plastic foam, as shown in Figure 2 (a). The magnetometer used in the proposed tracking system was a 3-axis Freescale magnetic sensor, MAG3110, which has a full-scale range of $\pm 1000 \mu \mathrm{T}$ and a sensitivity of $0.10 \mu \mathrm{T}$. A wooden platform, on which the magnetometer array (with 12 magnetometers) was mounted, is shown in Figure 2 (b). The magnetic flux density used in calculating the location and orientation of tracker should not include the earth magnetic field; therefore, the signal produced by the earth magnetic field should be filtered. In laboratory study of geohazards, signal from the earth magnetic field can be recorded at the very beginning and then be subtracted from the system considering the stability of earth magnetic field during an experiment.

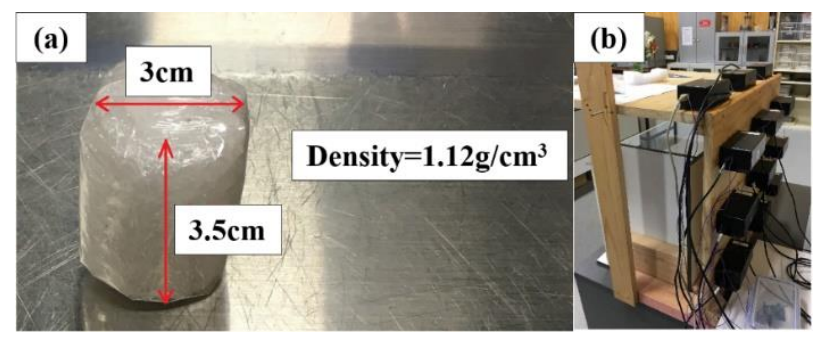

Fig. 2. (a) Permanent magnet covered by plastic foam; (b) wooden platform with 12 magnetometers

Since the effectiveness of a magnetic tracking system relies on an accurate detection of the magnetic field generated by the tracker, environmental interference should be evaluated carefully before the tracking system could be applied in a certain environment. Even though soil, as a main medium in geotechnical-orientated system, is believed to have little impact on tracking accuracy, some proofs are still considered necessary.

The control group was tested in which the tracker was exposed to air. For convenience, the tracker was set up to move in a rigid tube under a known path, which was fixed on the soil box, as shown in Figure 3(a). Test results derived from the magnetic tracking system showed that the $y$ - and $z$-coordinates of the movement stayed roughly constant at $y=-0.207 \mathrm{~m}$ and $z=-0.258 \mathrm{~m}$, respectively.

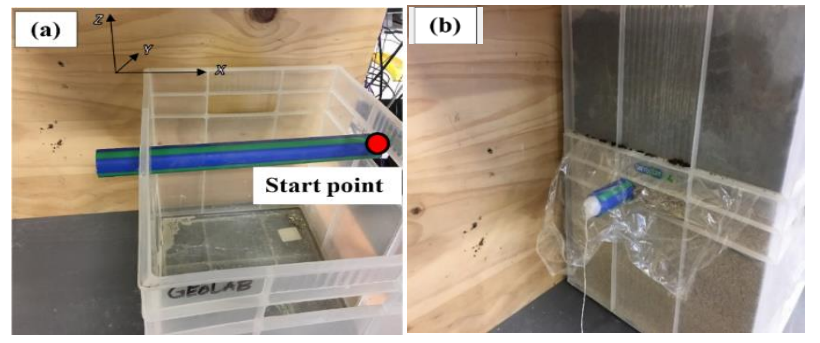

Fig. 3. (a) Tracker moving in a fixed tube as reference; (b) Tube buried in soil for the test group
For the test group, a soil box was filled with dry sand, which buried the tube completely. Another soil box filled with wet soil was placed right on top of the first box, as shown in Figure 3 (b). Results indicate that the trace of the tracker's movement was not changed that much, with $y=-0.209 \mathrm{~m}$ and $z=-0.259 \mathrm{~m}$, respectively. Thus, it can be observed that movements from both the control group and the test group have similar values on $z$ - as well as $y$-axis. Comparison of movements between the test group and control group projected on the $\mathrm{x}-\mathrm{z}$ surface is plotted in Figure 4, where no significant difference is observed. Because the tube was always fixed at the same location on the soil box, it can be concluded that the presence of soil (wet and dry) did not influence the general accuracy as expected.

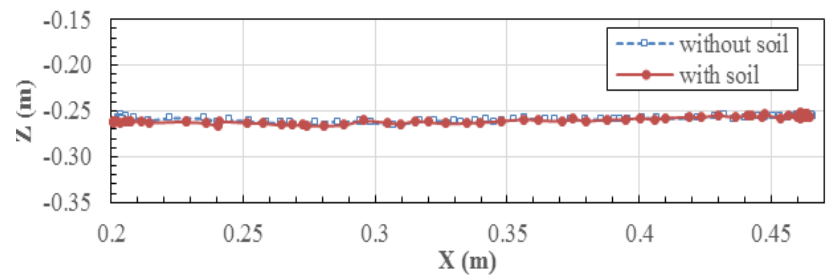

Fig. 4. Projections of movements on $x-z$ surface of test group (without soil) and control group (with soil).

\section{Multi-objective tracking}

In small scale geotechnical laboratory tests, it is usually necessary to track multiple objects, since the analysis of displacement field produced by a geohazard often requires displacement information obtained from different locations. For example, in the study of soilstructure interaction, an investigation of the different responses between isolated and adjacent structures can shed light on the mechanisms governing the performance of structures affected by geohazards, such as earthquake and liquefaction. In this case, multi-objective tracking is indispensable with trackers being deployed around both isolated and adjacent structures.

In this section, multi-objective tracking using the proposed magnetic tracking system is presented, with the first part discussing briefly the improved algorithm from the single objective tracking, and followed by tracking results and error analysis.

\subsection{Algorithm for multi-objective tracking}

Because each permanent magnet has 5 tracking unknowns with 3 indicating its location and 2 for its orientation, the minimum receiver (magnetometer) number is 5 for each magnet, or two 3-axis magnetic sensors. 12 3-axis Freescale magnetic sensors, MAG3110, were used for the previous verification tests, so it should be enough for the following multi-objective tracking.

Assuming $k(\geq 2)$ independent permanent magnets are tracked at the same time, their locations and orientations can be computed by solving high-order nonlinear equations given by Equations (5) - (7). 


$$
\begin{aligned}
& B_{x i}=\sum_{j=1}^{k} B_{T j}\left\{\frac{3\left[m_{j}\left(x_{i}-a_{j}\right)+n_{j}\left(y_{i}-b_{j}\right)+p_{j}\left(z_{i}-c_{j}\right)\right] \cdot\left(x_{i}-a_{j}\right)}{R_{i j}^{5}}-\frac{m_{j}}{R_{i j}^{3}}\right\} \\
& B_{y i}=\sum_{j=1}^{k} B_{T j}\left\{\frac{3\left[m_{j}\left(x_{i}-a_{j}\right)+n_{j}\left(y_{i}-b_{j}\right)+p_{j}\left(z_{i}-c_{j}\right)\right] \cdot\left(y_{i}-b_{j}\right)}{R_{i j}^{5}}-\frac{n_{j}}{R_{i j}^{3}}\right\} \\
& B_{z i}=\sum_{j=1}^{k} B_{T j}\left\{\frac{3\left[m_{j}\left(x_{i}-a_{j}\right)+n_{j}\left(y_{i}-b_{j}\right)+p_{j}\left(z_{i}-c_{j}\right)\right] \cdot\left(z_{i}-c_{j}\right)}{R_{i j}^{5}}-\frac{p_{j}}{R_{i j}^{3}}\right\}
\end{aligned}
$$

where $B_{x i}, B_{y i}$ and $B_{z i}$ are the $x, y$ and $z$ components of $\boldsymbol{B}_{\boldsymbol{i}}$, respectively. The rest variables are the same as indicated in Equation (1) - (4). In order to solve the high order nonlinear equations above, it is convenient to convert it into a constrained nonlinear optimization problem, which is described as follows:

Find $x^{*}$, a local minimizer for

$$
F(x)=\frac{1}{2} \sum_{i=1}^{m}\left(f_{i}(x)\right)^{2}=\frac{1}{2} \mathbf{f}(\mathbf{x})^{\mathrm{T}} \mathbf{f}(\mathbf{x})
$$

where $f_{i}: \mathcal{R}^{n} \rightarrow \mathcal{R}, i=1, \ldots, m$ are given functions, and $m \geq n=6 k$ ( $m$ is the number of different sets of input values, which equals to the total number of magnetometers used). $f_{i}(x)$ is the error between the measured data from magnetometers and the calculated one derived by substituting the assumed locations and orientations of each tracker into Equation (5) - (7). There are many nonlinear optimization algorithms widely used to solve the problem above. For example, Newton-Gauss method and Powell method have the advantage of time efficiency while bearing the disadvantage of requiring reasonable initial guesses. In this paper, a Particle Swarm Optimization (PSO) algorithm [9] was used to provide a set of initial guesses, which could be further used by an Interior-point algorithm [10] to produce solutions with acceptable accuracy. Equations for these algorithms are briefly discussed here.

First of all, the interior-point method is a type of nonlinear constrained optimization technique, which is widely used to minimize the objective functions subject to constraints, for example, Equation (8). The constraints in this case are described as follows:

$$
\begin{gathered}
m_{j}^{2}+n_{j}^{2}+p_{j}^{2}=1 \\
0 \leq a_{j} \leq 0.5 \\
-0.2 \leq b_{j} \leq 0 \\
-0.5 \leq c_{j} \leq 0
\end{gathered}
$$

where the variables are the same as indicated in Equations (1) - (4). This method uses barrier function and slack variables to convert the original inequalityconstrained problem to a sequence of equalityconstrained problems.

For each $\mu>0$, the converted problem is

$$
\min _{x, s} f_{\mu}(x, s)=\min _{x, s} F(x)-\mu \sum_{i}^{6} \ln \left(s_{j i}\right)
$$

$$
\begin{gathered}
m_{j}^{2}+n_{j}^{2}+p_{j}^{2}-1=0 \\
a_{j}-s_{j 1}=0 ; 0.5-a_{j}-s_{j 2}=0 \\
b_{j}+0.2-s_{j 3}=0 ;-b_{j}-s_{j 4}=0 \\
c_{j}+0.5-s_{j 5}=0 ; 0.1-c_{j}-s_{j 6}=0
\end{gathered}
$$

where $s_{j i}$ is the slack variable and $\mu \sum_{i}^{6} \ln \left(s_{j i}\right)$ is the barrier function. There are as many slack variables as there are inequality constraints. Besides, all the slack variables are restricted to be positive in order to keep the barrier function bounded, so that as $\mu$ decreases to zero, the minimum of $f_{\mu}$ should approach the minimum of the objective function. With equality constraints, the search direction can be found by Newton step. Iteration will stop when the convergence criteria are met.

Secondly, the PSO algorithm is used to generate an initial guess for the interior-point method to start iterating. In PSO, $N$ particles are assumed and generated, which are characterized by their positions and velocities. In an $n$-dimensional hyperspace (in this case $n=6 k$ ), the position of the particle $i(i=1,2 \ldots N)$, represented by

$$
x_{i}=\left(a_{i 1}, b_{i 1}, c_{i 1}, m_{i 1}, n_{i 1}, p_{i 1}, \ldots, a_{i k}, b_{i k}, c_{i k}, m_{i k}, n_{i k}, p_{i k}\right) \text {, }
$$

indicates the solution location in the hyperspace. Also, the movement of the particle is represented by its velocity

$$
v_{i}=\left(v_{a_{i 1}}, v_{b_{i 1}}, v_{c_{i 1}}, v_{m_{i 1}}, v_{n_{i 1}}, v_{p_{i 1}}, \ldots, v_{a_{i k}}, v_{b_{i k}}, v_{i l k}, v_{m_{i k}}, v_{n_{i k}}, v_{p_{i k}}\right)
$$

A set of historically best position for each particle is recorded as pbest $t_{i}$, and the global best position for all particles is gbest. Each particle $i(i=1,2 \ldots N)$ updates its location and velocity by:

$$
\begin{gathered}
\left.v_{i, t+1}=w v_{i, t}+c_{1} r_{1}\left(\text { pbest }_{i}-x_{i, t}\right)+c_{2} r_{2} \text { (gbest }-x_{i, t}\right) \\
x_{i, t+1}=x_{i, t}+v_{i, t+1}
\end{gathered}
$$

where $t$ is the iteration number; $w$ is the initial weight; $c_{1}$ and $c_{2}$ are two parameters representing the particle's confidence in its own best position and in the global one respectively; and $r_{1}$ and $r_{2}$ are two uniformly distributed random number between $[0,1]$. The algorithm will evaluate the value of the objective function in every iteration until the stop criterion is met.

subject to 


\subsection{Test results}

Two trackers were used to evaluate the performance of the proposed magnetic tracking system in multiobjective tracking, whose results were then compared with those derived from video images recorded when the two tackers were moving by hands on a hand-drawn ruler, as shown in Figure 5.

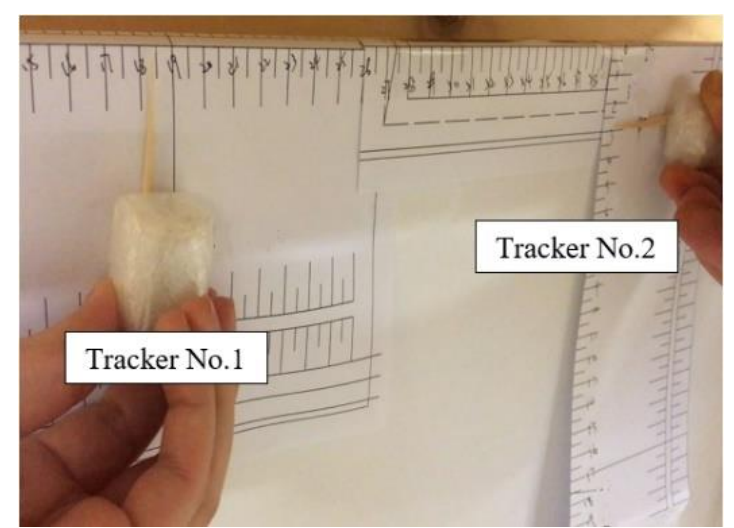

Fig.5. One of the photos extracted from video recording the two trackers moving on a hand-drawn ruler.

Results derived from the magnetic tracking system is shown in Figure 6, in which Tracker No. 1 (on the left) was moving horizontally and Tracker No. 2 (on the right) was moving roughly in the vertical direction.

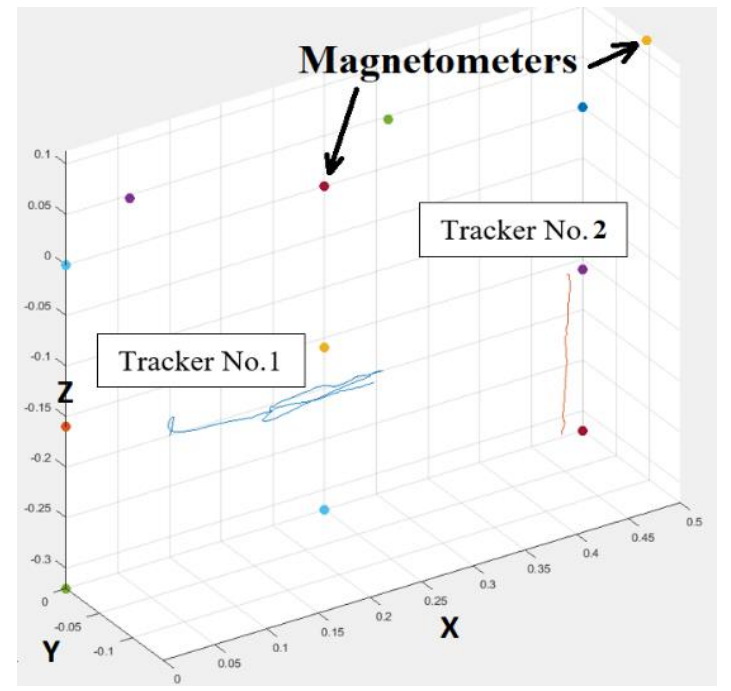

Fig.6. Movement paths of Tracker No. 1 (on the left) moving horizontally and Tracker No. 2 (on the right) moving vertically.

Comparisons between results from tracking system and video images are shown in Figure 7. Because video only recorded movements on the $\mathrm{x}-\mathrm{z}$ plane, $\mathrm{x}$-axis movements were considered dominant for Tracker No. 1 and were therefore compared, while comparison for Tracker No. 2 was conducted only along z-axis.

It can be seen from the results that the magnetic tracking system is able to detect the positions of the two magnets quite accurately. Errors were calculated by averaging the discrepancies between the data from video and magnetic tracking system at the times the samplings were taken. The magnetic tracking system is able to take
20 samples per one second with an average error of $\pm 2 \mathrm{~mm}$ under the circumstance in which: (1) the tracking plane is around $15-20 \mathrm{~cm}$ away from magnetometer array; and (2) the tracking plane is around $45-50 \mathrm{~cm}$ on each side.
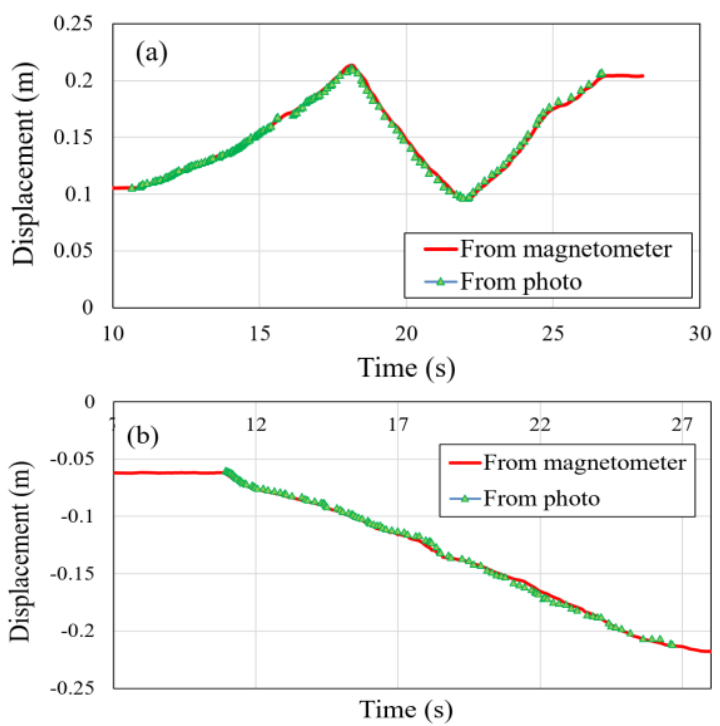

Fig. 7. (a) $X$-axis movement comparison from magnetometers and video recorded of Tracker No.1 (left); (b) $z$-axis movement comparison from magnetometers and video image recording of Tracker No.2 (right)

\section{Concluding remarks}

This paper presents a magnetic tracking system having the potential to use two permanent magnets as trackers to detect subsoil displacements due to geohazards. Results of verifications are also provided under common geotechnical environment with the presence of soil (both dry and wet). By increasing the sensitivity of magnetometers in the future, the sensing range of the tracking system could be increased accordingly in order to be adapted to different scales of geotechnical laboratory tests.

Being able to capture the displacement field of soil using multi-objective tracking can lead to a better understanding of the mechanisms involved in a geohazard. Besides, velocity as well as acceleration field are also of great importance in analysing the behaviours. Although velocity can be obtained by differentiating the displacement data (and also acceleration is the second derivative of displacement), errors may exist. It is always better to have another system providing additional information to not only verify the results but also improve the overall accuracy. For example, an inertial navigation system (INS) is able to first capture the information of acceleration and then calculate the displacement by integrating the acceleration two times. Therefore, since the integration processes lead to errors that grow with time, the displacements calculated are not always reliable compared to the directly obtained ones from the proposed magnetic tracking system. However, due to the fact that INS can provide relatively accurate measurements on acceleration, overall tracking accuracy 
could be increased by: (1) differentiating two times the displacements derived from magnetic tracking system; (2) fitting the calculated accelerations using data from INS; and (3) back calculating the displacements using the updated accelerations. As a result, a combination of the magnetic tracking system and INS may bring more possibilities into laboratory tests on geohazards.

The authors would like to acknowledge the assistance of staff of the Dept. of Electrical and Computer Engineering, University of Auckland, particularly Rob Champion, for the assembly of the magnetometers.

\section{References}

1. R.P. Orense, Geotechnical Hazards: Nature, Assessment and Mitigation, University of the Philippines Press, 510pp. (2003)

2. H. O. Baba, S. Peth, Large scale soil box test to investigate soil deformation and creep movement on slopes by Particle Image Velocimetry (PIV). Soil and Tillage Research, 125, 38-43 (2012).

3. W. A. Take, Thirty-Sixth Canadian Geotechnical Colloquium: Advances in visualization of geotechnical processes through digital image correlation. Canadian Geotechnical Journal, 52(9), 1199-1220 (2015).

4. H. McLeod, Field measurements of surface displacements from pipe bursting. Master's Thesis, Queens University, Ontario, Canada (2008).

5. V. Schlageter, P. A. Besse, R. S. Popovic, P. Kucera, Tracking system with five degrees of freedom using a 2D-array of Hall sensors and a permanent magnet. Sensors and Actuators A: Physical, 92(1-3), 37-42 (2001)

6. C. Hu, MQ-H. Meng, M. Mandal, Efficient linear algorithm for magnetic localization and orientation in capsule endoscopy. 2005 IEEE Engineering in Medicine and Biology 27th Annual Conference (2006).

7. H. Fialová, G. Maier, E. Petrovský, A. Kapička, T. Boyko, R. Scholger, MAGPROX Team, Magnetic properties of soils from sites with different geological and environmental settings. Journal of Applied Geophysics, 59(4), 273-283 (2006).

8. X. Chen, R.P. Orense, Development of magnetic tracking system for monitoring ground movements. Proceedings of 20th New Zealand Geotechnical Society Geotechnical Symposium, Napier, 23-26 November 2017, 8pp. (2017).

9. A. Wächter, L.T. Biegler. On the implementation of an interior-point filter line-search algorithm for large-scale nonlinear programming. Mathematical programming, 106(1), 25-57. (2006).

10. Q. Lin, J. Li, Z. Du, J. Chen, Z. Ming. A novel multi-objective particle swarm optimization with multiple search strategies. European Journal of Operational Research, 247(3), 732-744. (2015). 